\title{
Delayed cognitive deficits can be alleviated by calcium antagonist nimodipine by downregulation of apoptosis following whole brain radiotherapy
}

\author{
JING TONG ${ }^{1}$, JUAN LI ${ }^{2}$, QIU-SHI ZHANG ${ }^{1}$, JIAN-KAI YANG ${ }^{3}$, LEI ZHANG ${ }^{1}$, HAI-YING LIU ${ }^{1}$, \\ YING-ZI LIU $^{1}$, JIANG-WEI YUAN ${ }^{1}$, XU-MING SU $^{1}$, XUE-XIN ZHANG $^{1}$ and BAO-HUA JIAO ${ }^{3}$
}

Departments of ${ }^{1}$ Neurosurgery and ${ }^{2}$ Radiotherapy, The Fourth Hospital of Hebei Medical University, Shijiazhuang, Hebei 050011; ${ }^{3}$ Department of Neurosurgery, The Second Hospital of Hebei Medical University, Shijiazhuang, Hebei 050000, P.R. China

Received February 20, 2017; Accepted January 29, 2018

DOI: $10.3892 / \mathrm{ol} .2018 .8968$

\begin{abstract}
Radiation therapy is important for the comprehensive treatment of intracranial tumors. However, the molecular mechanisms underlying the pathogenesis of delayed cognitive dysfunction are not well-defined and effective treatments or prevention measures remain insufficient. In the present study, 60 adult male Wistar rats were randomly divided into three groups, which included a control, whole brain radiotherapy (WBRT) (single dose of $30 \mathrm{~Gy}$ of WBRT) and nimodipine (single dose of 30 Gy of WBRT followed by nimodipine injection intraperitoneally) groups. The rats were sacrificed 7 days or 3 months following irradiation. At 3 months, the Morris water maze test was used to assess spatial learning and memory function in rats. The results demonstrated that the WBRT group demonstrated a significantly impaired cognitive performance, decreased numbers of hippocampal Cornu Ammonis (CA)1 neurons and upregulated expression of caspase-3 in the dentate gyrus compared with those in the control and nimodipine groups. Reverse transcription-quantitative polymerase chain reaction analysis demonstrated that the WBRT group exhibited increased ratio of B-cell lymphoma 2 (Bcl-2)-associated $\mathrm{X}$ protein (Bax)/Bcl-2 compared with that in control and nimodipine groups on day 7 following irradiation. However, the WBRT group exhibited decreased levels of brain-derived neurotrophic factor (BDNF) compared with that in control and nimodipine groups at 3 months following brain irradiation. The levels of growth-associated protein 43 and amyloid precursor protein between the nimodipine group and WBRT group were not statistically significant. The
\end{abstract}

Correspondence to: Dr Bao-Hua Jiao, Department of Neurosurgery, The Second Hospital of Hebei Medical University, 215 Heping West Road, Shijiazhuang, Hebei 050000, P.R. China E-mail: jiaobh1215@163.com

Key words: whole brain radiation therapy, cognitive deficit, hippocampus, neuron apoptosis, calcium antagonist, rat present study demonstrated that neuron apoptosis may lead to delayed cognitive deficits in the hippocampus, in response to radiotherapy. The cognitive impairment may be alleviated in response to a calcium antagonist nimodipine. The molecular mechanisms involved in nimodipine-mediated protection against cognitive decline may involve the regulation of $\mathrm{Bax} / \mathrm{Bcl}-2$ and BDNF in the hippocampus.

\section{Introduction}

Radiotherapy is used for the treatment of malignant brain tumors, including high-grade gliomas, brain metastases, germinoma and medulloblastoma, and metastatic tumors of other sites, such as lung cancer $(1,2)$. Patients who received radiation therapy may develop delayed brain injury at 6 months or several years following therapy. Cognitive deficit is one of the complications of radiation therapy and mainly manifests as learning and memory disabilities (3). Previous studies demonstrated that glial cell injury $(3,4)$, cell aplasia $(3,5,6)$, vascular damage (7) and inflammation (8) may lead to radiation-induced brain injury. A previous study demonstrated that delayed cognitive deficits may be associated with apoptosis of hippocampal neurons in immature brain tissues following radiotherapy (9). However, the molecular mechanisms underlying the cognitive deficits following brain radiotherapy remain controversial and the clinical prevention and treatment approaches remain limited.

Calcium channels serve important functions in the central nervous system. Activation of calcium channels mediates signal transductions involved in physiological and pathological neuronal responses. Neurodegeneration induced by acute spinal cord injury and brain injury may be associated with oxidative stress induced by apoptosis and calcium influx $(10,11)$. Calcium channel blockers exhibit a protective effect against brain injury and cerebral ischemia. The underlying molecular mechanisms may involve the inhibition of calcium influx, decreased release of neurotransmitters and inhibition of apoptosis in neurons (12-14).

In the present study, a rat model of whole brain radiotherapy (WBRT) was established and calcium ion antagonist nimodipine was administered to evaluate its effects on delayed cognitive deficits following brain irradiation. 


\section{Materials and methods}

Animals. A total of 60 male, 10-week-old Wistar rats, with a mean body weight of $236 \pm 10 \mathrm{~g}$ were obtained from the Experimental Animal Center of Hebei Medical University (Hebei, China). Rats were housed in pairs in a light and temperature-controlled room $\left(22-24^{\circ} \mathrm{C}, 45-50 \%\right.$ humidity and $12 / 12 \mathrm{~h} \mathrm{light/dark}$ cycle) and received food and water ad libitum. Following anesthesia, rats were randomly divided into the following three groups: Control (rats did not undergo WBRT and were intraperitoneally injected with $2.5 \mathrm{ml} / \mathrm{kg}$ of saline daily for 7 consecutive days), WBRT (rats underwent WBRT and were intraperitoneally injected with $2.5 \mathrm{ml} / \mathrm{kg}$ of saline daily for 7 consecutive days) and nimodipine groups [rats underwent WBRT and were intraperitoneally injected with $2.5 \mathrm{ml} / \mathrm{kg}$ of calcium antagonist nimodipine $(0.5 \mathrm{mg} / \mathrm{kg} / \mathrm{day}$; Shandong Xinhua Pharmaceutical Co., Ltd., Shandong, China) daily following radiotherapy for 7 consecutive days]. Rats ( $n=10$ in each group) were sacrificed at 7 days or 3 months following WBRT. On day 7, fresh hippocampal tissues $(n=5$ in each group) were used for reverse transcription-quantitative polymerase chain reaction (RT-qPCR) analysis of $\mathrm{B}$-cell lymphoma 2 (Bcl-2)-associated $\mathrm{X}$ protein (Bax) and $\mathrm{Bcl}-2$ or for histological examination. For the 3-month timepoint, rats underwent the Morris water maze test to evaluate spatial learning and memory performance prior to sacrifice by decapitation. At three months, rats were randomly divided into two groups, one group $(n=5)$ was used in PCR of amyloid precursor protein (APP), brain-derived neurotrophic factor (BDNF) and growth-associated protein 43 (GAP-43); the other group $(n=5)$ was used in histological examination following the Morris water maze test. During the experimental procedures, the hair loss, skin damage and body weight gain rate [(measured weight value-basal weight value)/basal weight value] of rats was evaluated.

The procedures involving animals and their care were conducted in accordance with the Institutional Animal Care and Use Committee of Hebei Medical University (Hebei, China). The present study was approved by the Ethics Research Committee of the Second Hospital of Hebei Medical University (Hebei, China; approval no. 2015215).

Radiation. Rats were anesthetized with an intraperitoneal injection of chloral hydrate $(350 \mathrm{mg} / \mathrm{kg})$, placed on a prone position and irradiated using a 6-MeV linear accelerator (Varian Clinac iX; Varian Medical Systems, Palo Alto, CA, USA). Rats were exposed to 30 Gy radiation. Rats were irradiated from a vertical direction at a dose rate of $300 \mathrm{cGy} / \mathrm{min}$ and at a source-skin distance of $100 \mathrm{~cm}$. The size of radiation field was $2.0 \times 1.5 \mathrm{~cm}$ (eyes canthus to rear ears), whilst other parts of the body were sheltered by lead plates to protect against radiation (15). Rats in the control group received anesthesia only and did not undergo WBRT.

Cognitive function test. The cognitive function was evaluated using the Morris water maze test. Rats were placed at different starting positions in a circular pool (diameter, $120 \mathrm{~cm}$; height, $50 \mathrm{~cm}$ ) filled with water (depth, $37 \mathrm{~cm} ; 21-22^{\circ} \mathrm{C}$, made opaque by adding powdered milk to prevent the rats seeing the platform under the water). Rats were trained to locate a platform (diameter, $9 \mathrm{~cm}$; height, $36 \mathrm{~cm}$ ), which was submerged $1 \mathrm{~cm}$ below the water surface and located in a quadrant of the pool, using distant visual cues. These visual cues were present on the four walls surrounding the pool at a distance of $0.6 \mathrm{~m}$. For the training phase, each rat performed 5 acquisition trials/day (maximal swimming time $120 \mathrm{sec}$; $30 \mathrm{sec}$ on platform; inter-trial interval $30 \mathrm{~min}$ ) for 4 consecutive days. A different quadrant was selected each time as a point of entry. The rat from each of the three group rats used the same starting position for evaluation. All trials were recorded, and latency time, defined as the time to locate the platform, was used as a measure for spatial learning. The rats were allowed $60 \mathrm{sec}$ until they found the platform. If the rat failed to find the platform within $60 \mathrm{sec}$, the rat was placed on the platform and allowed to stay on it for $20 \mathrm{sec}$. The time taken for the rat to reach the platform was recorded. During the probe trial, the hidden platform was removed from the tank and the rats were allowed to swim freely for $60 \mathrm{sec}$. Latency to find the maze platform (sec) was recorded. All the trials were recorded and analyzed by an automated video-tracking system (JLBehv-M; version 2.1; Shanghai Jiliang Software Technology Co., Ltd., Xuhui, Shanghai, China).

Histological examination. For the histological examination, the rats were perfused with saline, followed by fixation with 4\% paraformaldehyde. The brain tissues (thickness, 1-4 mm behind the optic chiasma) were removed and stored in the same paraformaldehyde solution for $24 \mathrm{~h}$ and embedded in paraffin. Paraffin-embedded tissue samples were cut into $5-\mu \mathrm{m}$ thick sections. A total of five nonconsecutive sections were used for histological examination. Slices were incubated at $60^{\circ} \mathrm{C}$ for $30 \mathrm{~min}$, then cooled, cleared with xylene twice for 5 min each, dehydrated with 100, 95, 90 and $85 \%$ ethanol each for $5 \mathrm{~min}$ and then with 80 and $70 \%$ ethanol each for 3 min. Furthermore, slices were rinsed with distilled water for $1 \mathrm{~min}$, stained with $0.1 \%$ cresyl violet dye in an oven at $37^{\circ} \mathrm{C}$ for $30 \mathrm{~min}$, rinsed with water for $8 \mathrm{~min}$ and rapidly separated with $95 \%$ ethanol. Following this, sections were incubated in anhydrous alcohol and xylene twice for $5 \mathrm{~min}$ each prior to mounting. The slices were observed and imaged with a Nikon ECLIPSE 50i light microscope at magnification, x400 (Nikon Corporation, Tokyo, Japan). The neuronal density was determined by the average number of surviving hippocampal Cornu Ammonis (CA) 1 in each 1-mm section, with three sections of bilateral hippocampal slices (16).

Detection of apoptosis of neurons in the dentate gyrus of the hippocampus was evaluated using immunohistochemical staining for caspase-3. Prior to staining, the sections were incubated for $30 \mathrm{~min}$ at $65^{\circ} \mathrm{C}$ and deparaffinized with xylene and graded series of ethanol (100, 95, 90 and $85 \%$ ethanol each for $5 \mathrm{~min}$ and then with 80 and $70 \%$ ethanol each for $3 \mathrm{~min}$ ). Antigen retrieval was performed by boiling the sections in $10 \mathrm{mM}$ sodium citrate buffer (AM9763; pH 6.0; Thermo Fisher Scientific Inc., Waltham, MA, USA) for $10 \mathrm{~min}$ at room temperature. The sections were pre-treated with $10 \mu \mathrm{g} / \mathrm{ml}$ proteinase K (SC-473603; Santa Cruz Biotechnology, Inc., Dallas, TX, USA) for $10 \mathrm{~min}$ at room temperature prior to incubation with $4 \%$ normal goat serum (cat. no. ab7481; Abcam, Cambridge, USA) at room temperature for $1 \mathrm{~h}$. The primary antibody against active 


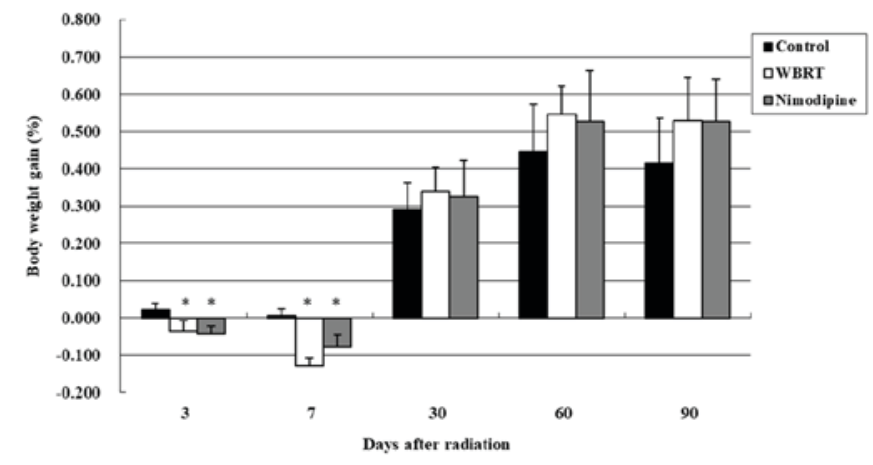

Figure 1. Changes in body weight among the control, WBRT and nimodipine groups on day $3,7,30,60$ and 90 following radiation. There were significant differences in body weight gain between rats in the WBRT $(\mathrm{P}<0.01)$ and nimodipine $(\mathrm{P}<0.01)$ group, compared with the control group at day 3 and 7 after brain radiation. WBRT, whole brain radiotherapy. ${ }^{*} \mathrm{P}<0.05$, vs. control.

caspase-3 (1:100; BS1518; Bioworld Technology, Inc., St. Louis Park, MN, USA) was incubated in a humidified box at $4^{\circ} \mathrm{C}$ overnight, followed by an $1 \mathrm{~h}$ incubation at room temperature with a biotinylated secondary goat anti-rabbit antibody (1:400; SC-2040; Santa Cruz Biotechnology, Inc.). 3,3'-diaminobenzidine (D12384; Sigma-Aldrich; Merck KGaA, Darmstadt, Germany) to visualize the antigen; following this, the sections were counterstained with Hematoxylin (H9627; Sigma-Aldrich; Merck KGaA) 5 min at room temperature, dehydrated in ethanol $(80,95,95$, 100 and $100 \%$, each for $3 \mathrm{~min}$ ) and cleared in xylene. The sections were mounted and the caspase-3-positive cells were observed and counted under a Nikon ECLIPSE 50i light microscope at magnification, $\mathrm{x} 400$.

$R T-q P C R$. Hippocampal tissues from the rats were removed at different time points, which included seven days and three months following WBRT. To extract total RNA, the frozen hippocampi were homogenized in $1.0 \mathrm{ml} \mathrm{TRIzol}{ }^{\circledR}$ (cat. no. 15596-026; Thermo Fisher Scientific Inc.). Following adding $200 \mu \mathrm{l}$ chloroform and placing on ice for $5 \mathrm{~min}$, homogenates were centrifuged $\left(12,000 \mathrm{x}\right.$ g for $15 \mathrm{~min}$ at $\left.4^{\circ} \mathrm{C}\right)$ and the supernatant was precipitated with $1.0 \mathrm{ml}$ isopropanol, then placed on ice for $10 \mathrm{~min}$ and centrifuged $(12,000 \mathrm{x} \mathrm{g}$ for $10 \mathrm{~min}$ at $\left.4^{\circ} \mathrm{C}\right)$. The supernatant fluid was removed, $400 \mu \mathrm{l}$ $75 \%$ ethanol was added and then the tube was centrifuged $\left(12,000 \mathrm{x} \mathrm{g}\right.$ for $5 \mathrm{~min}$ at $\left.4^{\circ} \mathrm{C}\right)$. Following this the supernatant fluid was removed and dried on filter paper. The powder was dissolved by $0.1 \%$ diethylpyrocarbonate. The RNA was reverse transcribed to cDNA using a RevertAid First Strand cDNA synthesis kit (K1622; Thermo Fisher Scientific Inc.), following the manufacturer's protocol. RT-qPCR reactions were performed using Platinum SYBR-Green qPCR SuperMix-UDG (CS11733038; Invitrogen; Thermo Fisher Scientific, Inc.). For the rats in the seven days group, the following primer pairs were used: Bax forward, GGCGAA TTGGCGATGAACTG and reverse, ATGGTTCTGATC AGCTCGGG; and Bcl-2 forward, GGGGCTACGAGTGGG ATACT and reverse, GACGGTAGCGACGAGAGAAG. The level of expression of these two tested genes was normalized to the level of expression of the $\beta$-actin gene forward, GGA GATTACTGCCCTGGCTCCTA and reverse, GACTCA
TCGTACTCCTGCTTGCTG. For the rats in the three months group, the following primer pairs were used: APP forward, TCGTGATTCCTTACCGGTGC and reverse, ATC GATGCTGTCGCTCTCCT; BDNF forward, GGTCACAGT CCTGGAGAAAG and reverse, GTCTATCCTTATGAA CCGCC; and GAP-43 forward, GCTAGCTTCCGTGGA CACAT and reverse, ACCATCAGCAACGGGAGCAT. GAPDH forward, AGGTGAAGGTCGGAGTCAACG and reverse, AGGGGTCATTGATGGCAACA was used as the internal control. PCR reactions were performed with initial denaturation at $95^{\circ} \mathrm{C}$ for $5 \mathrm{~min}$, followed by 40 cycles at $95^{\circ} \mathrm{C}$ for $30 \mathrm{sec}, 58^{\circ} \mathrm{C}$ (for $\mathrm{Bax}$ ), $56^{\circ} \mathrm{C}$ (for $\mathrm{Bcl}-2$ and $\mathrm{BDNF}$ ), $59^{\circ} \mathrm{C}$ (for GAP-43) and $57^{\circ} \mathrm{C}$ (for APP and GAPDH) for $30 \mathrm{sec}$ and $72^{\circ} \mathrm{C}$ for $45 \mathrm{sec}$. Real-time PCR was performed using an Mx3000P Real Time PCR system (Agilent, Technologies, Inc., Santa Clara, CA, USA). The quantification cycle (Cq) of target genes was normalized to that of internal control. mRNA levels in WBRT or nimodipine group animals were calculated after normalizing the quantification cycle to internal control expression, and the $2^{-\Delta \Delta \mathrm{Cq}}$ method was used to calculate the degree of expression (17).

Statistical analysis. Data were analyzed using SPSS software (version 20.0; IBM Corp., Armonk, NY, USA). Data are expressed as the mean \pm standard deviation. One-way analysis of variance was performed followed by the least significant difference test. $\mathrm{P}<0.05$ was considered to indicate a statistically significant difference.

\section{Results}

Evaluation of body weight following WBRT. A total of 60 rats were divided into three groups: control, WBRT and nimodipine groups and the body weight gain was evaluated on day 3, 730 , 60 and 90 following irradiation (Fig. 1). On day 3, body weight gain rates were $0.02 \pm 0.02,-0.04 \pm 0.03$ and $-0.04 \pm 0.02$ in the control, WBRT and nimodipine groups, respectively. One-way ANOVA revealed that the differences in the body weight gain were statistically significant $(\mathrm{P}=0.001)$ among the three groups. The results demonstrated WBRT and nimodipine groups lost weight, compared with control groups, three days after brain radiation and the differences were statistically significant $(\mathrm{P}=0.001)$. However, no differences in the body weight gain were observed between the nimodipine and WBRT groups on day $3(\mathrm{P}=0.649)$. There remained a significant difference in body weight gain between rats in the nimodipine $(\mathrm{P}=0.007)$ or WBRT $(\mathrm{P}<0.001)$ groups, compared with the control group, following seven days; however, no differences in the body weight gain were observed among the three groups following 1, 2 and 3 months of radiotherapy (Fig. 1).

Treatment with nimodipine improves cognitive function following WBRT. During irradiation, the physiological appearance and behavior of rats was monitored. Rats of WBRT and nimodipine groups exhibited hair loss and skin damage one week after brain radiation.

The cognitive function of rats was evaluated using a Morris water maze test three months after brain radiation. The results of the pre-training (Day 0) did not differ among the three groups $(\mathrm{P}=0.397)$. On day 1 , there was a significant 
difference in latency to the platform among the three groups $(\mathrm{P}<0.05)$. Rats from the control $(\mathrm{P}=0.003)$ and nimodipine groups $(\mathrm{P}=0.019)$ have an improved performance, compared with the WBRT group. On days 2, 3 and 4, no significant differences in latency to the platform were observed among the three groups $(\mathrm{P}>0.05)$ (Fig. 2A). The average latency was $28.80 \pm 18.13,35.76 \pm 20.94$ and $30.48 \pm 17.83$ for control, WBRT and nimodipine groups, respectively.

During the probe trial, the ratio of platform crossing/total distance in the WBRT $(24.33 \pm 1.17 \%)$ group was smaller, compared with the control $(29.63 \pm 2.25 \% ; \mathrm{P}=0.001)$ and nimodipine $(28.24 \pm 2.36 \% ; \mathrm{P}=0.009)$ groups. The data revealed that the differences in platform crossing times among the three groups were statistically significant $(\mathrm{P}=0.003)$. These results demonstrated that the cognitive function of rats in the WBRT group was poorer compared with that in the control and treatment with nimodipine improved the cognitive function of rats (Fig. 2B).

Decreased numbers of neurons following WBRT. Neuronal survival was assessed using Nissl staining. The results revealed that the neurons in the CA1 region of the hippocampus exhibited intact morphology in the control group (Fig. 3A). The neurons in the WBRT group appeared to be decreased, exhibited a 'fuzzy' appearance and degeneration (Fig. 3B). The numbers of neurons was decreased in the CA1 region in the WBRT group $\left(85.3 \pm 22.9 / \mathrm{mm}^{2}\right)$ compared with that in the control group $\left(183.2 \pm 27.6 / \mathrm{mm}^{2}\right)$. The number of neurons in CA1 region was increased in the nimodipine group (Fig. 3C; $128 \pm 19.5 / \mathrm{mm}^{2}$ ) compared with that in the WBRT group. The differences in the numbers of neurons among these three groups were statistically significant $(\mathrm{P}<0.01$; Fig. 3). These results suggest that the morphology and survival number of neurons in the hippocampus were affected following WBRT (Fig. 3).

Cell apoptosis is increased following WBRT. Immunohistochemical staining of caspase-3 (Fig. 4), revealed that the expression of caspase- 3 was increased in the neurons in the hippocampal dentate gyrus area in the WBRT group (Fig. 4B) compared with that in the control group (Fig. 4A). However, the expression of caspase-3 decreased in the hippocampal dentate gyrus area in the nimodipine group (Fig. 4C) compared with that in the WBRT group (Fig. 4B).

RT-qPCR analysis was performed to assess the Bax/Bcl-2 ratio among the three groups. The differences in the Bax/Bcl-2 ratio among these three groups were statistically significant $(\mathrm{P}=0.002)$. The results demonstrated that the $\mathrm{Bax} / \mathrm{Bcl}-2$ ratio was upregulated in the WBRT group $(0.62 \pm 0.16)$, compared with that in the control group $(0.39 \pm 0.14 ; \mathrm{P}=0.001$; Fig. 5), suggesting that cell apoptosis may increase in response to irradiation. Additionally, the $\mathrm{Bax} / \mathrm{Bcl}-2$ ratio in the nimodipine group $(0.47 \pm 0.10, \mathrm{P}=0.192)$ was marginally increased, compared with control group but significantly decreased, compared with the WBRT group ( $\mathrm{P}=0.016$; Fig. 5).

Expression levels of BDNF, APP and GAP-43 following $W B R T$. The expression of genes associated with cognitive function, including BDNF, GAP-43 and APP (18-21) was assessed using RT-qPCR. The results demonstrated that the expression
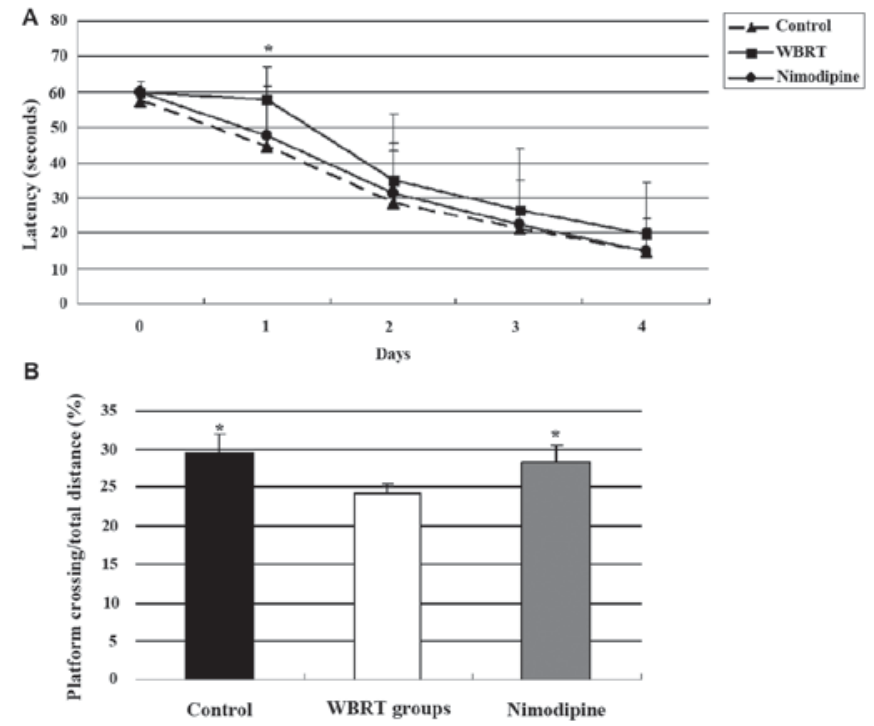

Figure 2. Evaluation of cognitive function following WBRT. (A) Latency to reach the platform in the Morris water maze. Rats in the control $(\mathrm{P}=0.003)$ and nimodipine group $(\mathrm{P}=0.019)$ had an improved performance, compared with WBRT group on day 1 following brain radiation. (B) The ratio of the platform crossing/total distance decreased and the memory ability of rats declined in the WBRT group. WBRT, whole brain radiotherapy. "P<0.05 vs. WBRT.

levels of BDNF $(5.13 \pm 0.46)$ and GAP-43 (4.57 \pm 0.35$)$ were decreased in the hippocampus of the WBRT group, compared with those in the control group [6.93 $\pm 0.61 \quad(\mathrm{P}=0.002)$; $5.89 \pm 1.20(\mathrm{P}=0.024)$, respectively]. However, the expression levels of BDNF increased in the nimodipine group (7.18 \pm 1.00$)$ compared with that in the WBRT group $(\mathrm{P}<0.01$; Fig. 6). The expression levels of GAP-43 between the nimodipine (5.05 \pm 0.63$)$ and WBRT group were not statistically significant $(\mathrm{P}=0.360)$. Additionally, the expression levels of APP $(4.74 \pm 1.43)$ were significantly increased in the WBRT group compared with that in the control group $(3.02 \pm 0.30 ; \mathrm{P}=0.016)$. However, nimodipine treatment did not affect significantly the expression levels of APP $(4.25 \pm 0.82)$ compared with that in the WBRT group ( $\mathrm{P}=0.441$; Fig. 6$)$.

\section{Discussion}

The improvement of the comprehensive treatment of tumors prolonged the survival time of patients with malignant brain tumors. Cognitive defects caused by radiotherapy have attracted increased attention $(22,23)$. In the present study, a rat model of WBRT was established. Additionally, the molecular mechanism underlying delayed cognitive deficits and the effects of calcium antagonists following radiotherapy were investigated.

The results of the present study demonstrated that rats developed learning and memory dysfunction at 3 months following a single 30 Gy dose of WBRT. This model may be used to observe delayed brain injury. Previous studies investigated strategies to optimize animal models for radiotherapy. Shi et al (24) used fractionated radiotherapy with a total of 45 Gy delivered as nine fractions of 5 Gy twice per week for 4.5 weeks and observed for 12 months. Compared to sham-irradiated rats, the irradiated rats demonstrated impaired MWM performance. Semmler et al (25) suggested a model whereby 

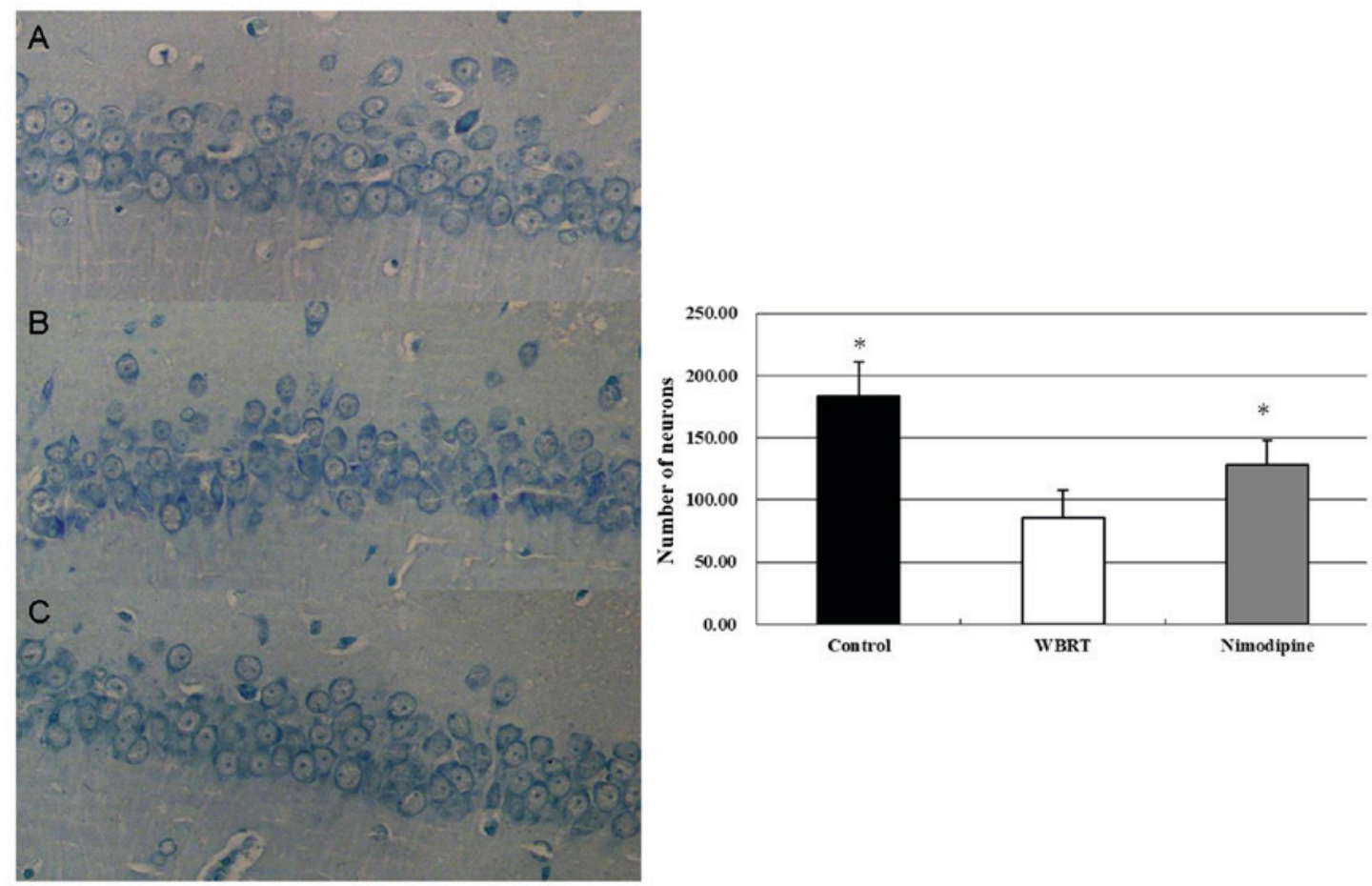

Figure 3. Decreased numbers of neurons following WBRT. The results by Nissl staining using a microscope (magnification, x400) revealed that the neurons in the CA1 region exhibited intact morphology in the control (A) but the neurons in the WBRT (B) appeared to be decreased and exhibited degeneration. However, nimodipine (C) group reserved more intact neurons compared with WBRT. The graph revealed that the number of neurons in hippocampal DG region in WBRT was significantly decreased compared with control $(\mathrm{P}<0.01)$ or nimodipine $(\mathrm{P}<0.01)$ group. $\mathrm{P}<0.05$ vs. WBRT. WBRT, whole brain radiotherapy; DG, dentate gyrus.

A

B

C

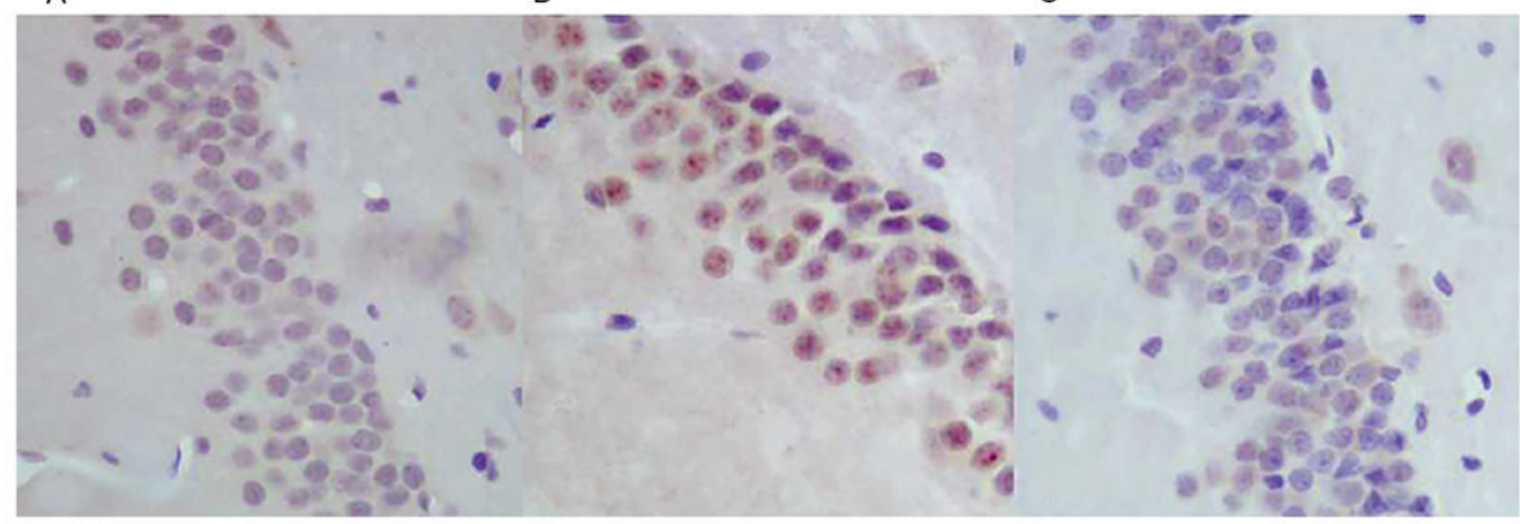

Figure 4. Caspase-3 staining in the hippocampal DG region. The expression levels of caspase-3 in (A) control, (B) WBRT and (C) nimodipine groups. The results demonstrated that the expression of caspase- 3 increased in the hippocampal CA1 region in the WBRT group compared with that in control and nimodipine groups. WBRT, whole brain radiotherapy; CA, Cornu Ammonis (magnification, x400).

rats were irradiated for four consecutive days with a dose of 5 or 10 Gy and were observed $\leq 6$ weeks following irradiation. After two weeks following irradiation, the rats demonstrated decreased open-field activity but no cognitive deficit as indicated by latencies in the Morris water maze test. After six weeks following irradiation, no group demonstrated alterations in histopathology, such as vascular changes, demyelination or white matter necrosis. An increased cumulative dosage and dose per fraction was used in this model to achieve a higher degree of radiation-induced toxicity. In the present study, rats exhibited learning and memory deficits at 3 months following a single 30 Gy dose of WBRT. Nissl staining demonstrated that the numbers of neurons in the CA1 region of the hippocampus decreased and the morphology of the cells changed following WBRT. These results confirm the efficient establishment of the delayed brain injury model following WBRT. The animal model employed in the present study did not require repeated anesthesia procedures due to a single radiation course and the success rate of delayed brain injury was increased, compared with animal models established in previous studies.

Increased apoptosis of neurons in the hippocampus may be a possible molecular mechanism underlying the impairment in learning and memory following WBRT $(26,27)$. Apoptosis serves a key function in several types of brain injury including 
ischemic cerebral infarction, traumatic brain injury, neonatal hypoxic encephalopathy and toxic encephalopathy. Previous studies have also revealed that the apoptosis of neurons may occur following brain radiotherapy (28-30). The present study revealed that the numbers of neurons in the CA1 region of the hippocampus decreased and the morphology of the cells changed following WBRT. Furthermore, the expression levels of caspase-3 were upregulated in the dentate gyrus of the hippocampus following WBRT, suggesting that apoptosis of hippocampal neurons was increased following WBRT.

According to previous studies, hippocampus may be associated with the learning and memory function of the brain, and with spatial memory $(31,32)$. Complete or partial hippocampal damage may lead to spatial learning and memory impairment (33). A previous study suggested that increased neurogenesis in the hippocampal dentate gyrus induced by electrical stimulation of the anterior thalamus may improve memory function (34). According to these results, it was hypothesized that the increase in apoptosis and the decrease in the number of neurons in the hippocampus following WBRT may lead to learning and memory dysfunction.

Administration of a calcium antagonist nimodipine may partly inhibit the apoptosis of neurons in the hippocampus and improve cognitive function following WBRT. Previous studies on cerebral ischemia, brain injury, chronic alcoholic encephalopathy, subarachnoid hemorrhage or cerebral hemorrhage demonstrated that calcium antagonists may exert a protective effect on brain function $(12,35-38)$. The expression levels of calcium-associated genes decreased following cerebral ischemia, leading to inhibition of L-type calcium channels, which in turn may induce post-ischemic neuronal death. Calcium antagonists may increase the survival of neurons in the hippocampal CA1 region and improve learning and memory function following cerebral ischemia $(14,39)$. The results of the Morris water maze test demonstrated that rats in the nimodipine group spent less time finding the hidden platform and had increased platform crossing, compared with the WBRT group. These results suggest that nimodipine may improve the learning and memory function of rats following brain radiotherapy. Nissl staining and immunohistochemistry analysis revealed that nimodipine may increase the number of neurons in the CA1 region and partially downregulated the expression of caspase- 3 in the dentate gyrus region of the hippocampus of rats following radiotherapy. These results suggested that the application of nimodipine may partially inhibit apoptosis of neurons in the hippocampus of rats following brain radiotherapy.

The molecular mechanism whereby nimodipine may inhibit the apoptosis of hippocampal neurons following radiotherapy may involve the regulation of $\mathrm{BDNF}$ and $\mathrm{Bax} / \mathrm{Bcl}-2$ ratio. BDNF and $\mathrm{Bcl}-2$ are associated with the survival of neurons (40-42). Neuronal apoptosis is correlated with the interactions between apoptosis promoting factors and anti-apoptotic factors. During apoptosis, Bcl-2 family members may interact with apoptosis-promoting factors and regulate the release of cytochrome $\mathrm{C}$ from mitochondria, inducing cell death (43). Bax is the main apoptosis-promoting factor in the nervous system that interacts with the Bcl-2 family members. Bcl-2-dependent and Bax-dependent apoptosis is associated with the regulation of caspase-3 in the brain $(43,44)$. A previous study demonstrated

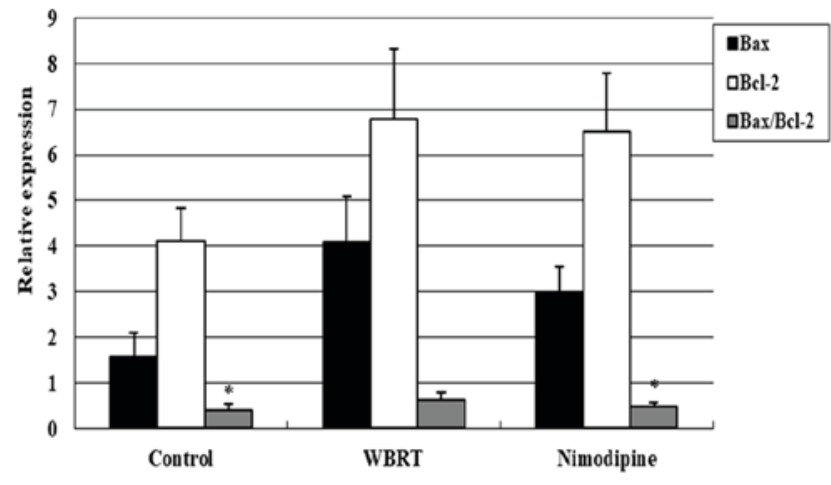

Figure 5. Expression of Bax and Bcl-2 following WBRT. The Bax/Bcl-2 ratio in the WBRT hippocampus was increased, compared with the control $(\mathrm{P}=0.001)$ and nimodipine groups $(\mathrm{P}=0.016)$ on day 7 after brain radiation. WBRT, whole brain radiotherapy; Bcl-2, B-cell lymphoma 2; Bax, Bcl-2-associated $\mathrm{X}$ protein. ${ }^{*} \mathrm{P}<0.05$, vs. WBRT.

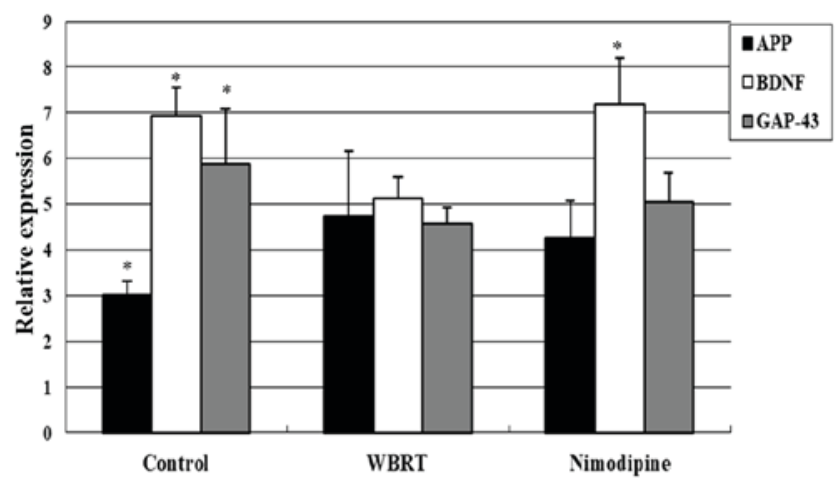

Figure 6. Expression levels of APP, BDNF and GAP-43 following WBRT. Expression levels of BDNF $(\mathrm{P}=0.002)$ and GAP-43 $(\mathrm{P}=0.024)$ decreased in the hippocampus of rats in the WBRT group compared with that in the control group. The expression level of BDNF increased in the nimodipine group, compared with the WBRT group $(\mathrm{P}<0.01)$. Expression of APP was upregulated in the WBRT group, compared with that in the control group; however, nimodipine treatment did not significantly downregulate APP expression, compared with the WBRT group $(\mathrm{P}=0.441)$. WBRT, whole brain radiotherapy; APP, amyloid precursor protein; BDNF, brain-derived neurotrophic factor; GAP-43, growth-associated protein 43 . ${ }^{*} \mathrm{P}<0.05$, vs. WBRT.

that Bax-deficient mice with brain injury exhibited increased numbers of neural progenitor cells in the dentate gyrus and improved remodeling of the hippocampus (45). In the present study, RT-qPCR analysis indicated that nimodipine may decrease the ratio of $\mathrm{Bax} / \mathrm{Bcl}-2$ and increase the expression levels of BDNF following WBRT. These results suggest that the calcium ion antagonist may downregulate the Bax/Bcl-2 ratio by inhibiting the release of Bax, upregulating the expression of BDNF, inhibiting apoptosis of hippocampal neurons following radiotherapy, thus improving cognitive function.

To conclude, the present study indicated that delayed cognitive deficits may occur in rats following whole brain radiation, and the application of calcium ion antagonist may improve cognitive function following radiotherapy. The molecular mechanism underlying the effects of nimodipine following WBRT may involve the inhibition of the apoptosis of neurons in the hippocampus. Nevertheless, further studies are required to elucidate the duration and dose of calcium ion antagonist treatment and provide more reliable and comprehensive data 
for the study of cognitive deficits, clinical prevention and treatment.

\section{Acknowledgements}

Not applicable.

\section{Funding}

The study is supported by a grant of Support Plan Project sponsored by Hebei Science and Technology Department (grant no. 122777101) and grant of Project of Science and Technology activities for returning scientists in Hebei Province (grant no. C2012003029).

\section{Availability of data and materials}

All data generated or analyzed during this study are included in this published article.

\section{Authors' contributions}

JT, XXZ, BHJ participated in the conception and design of the study. JL, QSZ, JKY, JWY and XMS conducted the experiments. LZ, HYL, YZL acquired and analyzed the data. JT and BHJ drafted and revised the manuscript. All authors read and approved the final manuscript.

\section{Ethical approval and consent to participate}

The procedures involving animals and their care were conducted in accordance with the Institutional Animal Care and Use Committee of Hebei Medical University (Hebei, China). The present study was approved by the Ethics Research Committee of the Second Hospital of Hebei Medical University (Hebei, China; approval no. 2015215).

\section{Patient consent for publication}

Not applicable.

\section{Competing interests}

The authors declare that they have no competing interests.

\section{References}

1. Surma-aho O, Niemelä M, Vilkki J, Kouri M, Brander A, Salonen O, Paetau A, Kallio M, Pyykkönen J and Jääskeläinen J: Adverse long-term effects of brain radiotherapy in adult low-grade glioma patients. Neurology 56: 1285-1290, 2001.

2. Blomstrand M, Brodin NP, Munck Af Rosenschöld P, Vogelius IR, SHnchez Merino G, Kiil-Berthlesen A, Blomgren K, Lannering B, Bentzen SM and Björk-Eriksson T: Estimated clinical benefit of protecting neurogenesis in the developing brain during radiation therapy for pediatric medulloblastoma. Neuro Oncol 14: 882-889, 2012.

3. Greene-Schloesser D, Moore E and Robbins ME: Molecular pathways: Radiation-induced cognitive impairment. Clin Cancer Res 19: 2294-2300, 2013

4. Sano K, Morii K, Sato M, Mori H and Tanaka R: Radiation-induced apoptosis and injury of oligodendrocytes on neonatal rat brains. Neurol Med Chir (Tokyo) 40: 495-500, 2000 .
5. Naylor AS, Bull C, Nilsson MK, Zhu C, Björk-Eriksson T, Eriksson PS, Blomgren K and Kuhn HG: Voluntary running rescues adult hippocampal neurogenesis after irradiation of the young mouse brain. Proc Natl Acad Sci USA 105: 14632-14637, 2008 .

6. Balentova S and Adamkov M: Molecular, cellular and functional effects of radiation-induced brain injury: A review. Int J Mol Sci 16: 27796-27815, 2015.

7. Brown WR, Blair RM, Moody DM, Thore CR, Ahmed S, Robbins ME and Wheeler KT: Capillary loss precedes the cognitive impairment induced by fractionated whole-brain irradiation: A potential rat model of vascular dementia. J Neurol Sci 257: 67-71, 2007.

8. Jenrow KA, Brown SL, Lapanowski K, Naei H, Kolozsvary A and Kim JH: Selective inhibition of microglia-mediated neuroinflammation mitigates radiation-induced cognitive impairment. Radiat Res 179: 549-556, 2013.

9. Huo K, Sun Y, Li H, Du X, Wang X, Karlsson N, Zhu C and Blomgren K: Lithium reduced neural progenitor apoptosis in the hippocampus and ameliorated functional deficits after irradiation to the immature mouse brain. Mol Cell Neurosci 51: 32-42, 2012.

10. Vosler PS, Brennan CS and Chen J: Calpain-mediated signaling mechanisms in neuronal injury and neurodegeneration. Mol Neurobiol 38: 78-100, 2008.

11. Nazırog ${ }^{\smile}$ lu M, Senol N, Ghazizadeh V and Yürüker V: Neuroprotection induced by $\mathrm{N}$-acetylcysteine and selenium against traumatic brain injury-induced apoptosis and calcium entry in hippocampus of rat. Cell Mol Neurobiol 34: 895-903, 2014.

12. Choi SK, Lee GJ, Choi S, Kim YJ, Park HK and Park BJ: Neuroprotective effects by nimodipine treatment in the experimental global ischemic rat model: Real time estimation of glutamate. J Korean Neurosurg Soc 49: $1-7,2011$.

13. Bullock R, Zauner A, Woodward J and Young HF: Massive persistent release of excitatory amino acids following human occlusive stroke. Stroke 26: 2187-2189, 1995.

14. Hu HH, Li SJ, Wang P, Yan HC, Cao X, Hou FQ, Fang YY, Zhu XH and Gao TM: An L-Type Calcium Channel Agonist, Bay K8644, Extends the Window of intervention against ischemic neuronal injury. Mol Neurobiol 47: 280-289, 2013.

15. Chen Y, Sun AM, Chen ZX, Liu Y, Chen LH and Yuan YW: Ginsenoside Rg1 protects rat hippocampal neurons from radiation injury by regulating NOS activity. Nan Fang Yi Ke Da Xue Xue Bao 30: 1522-1555, 2010 (In Chinese).

16. Zhang DS, Liu YL, Zhu DQ, Huang XJ and Luo CH: Point application with Angong Niuhuang sticker protects hippocampal and cortical neurons in rats with cerebral ischemia. Neural Regen Res 10: 286-291, 2015.

17. Livak KJ and Schmittgen TD: Analysis of relative gene expression data using real-time quantitative PCR and the 2(-Delta Delta C(T)) method. Methods 25: 402-408, 2001.

18. Bekinschtein P, Kent BA, Oomen CA, Clemenson GD, Gage FH, Saksida LM and Bussey TJ: Brain-derived neurotrophic factor interacts with adult-born immature cells in the dentate gyrus during consolidation of overlapping memories. Hippocampus Aug 24: 905-911, 2014.

19. Jeon YK and Ha CH: The effect of exercise intensity on brain derived neurotrophic factor and memory in adolescents. Environ Health Prev Med 22: 27, 2017.

20. Holahan MR: A shift from a pivotal to supporting role for the growth-associated protein (GAP-43) in the coordination of axonal structural and functional plasticity. Front Cell Neurosci 11: 266, 2017.

21. Preat T and Goguel V: Role of drosophila amyloid precursor protein in memory formation. PLoS One 12: 142, 2016.

22. Ganau L, Paris M, Ligarotti GK and Ganau M: Management of gliomas: Overview of the latest technological advancements and related behavioral drawbacks. Behav Neurol 2015: 862634, 2015.

23. Brown PD, Jaeckle K, Ballman KV, Farace E, Cerhan JH, Anderson SK, Carrero XW, Barker FG II, Deming R, Burri $\mathrm{SH}$, et al: Effect of radiosurgery alone vs radiosurgery with whole brain radiation therapy on cognitive function in patients with 1 to 3 brain metastases: A randomized clinical trial. JAMA 316: 401-409, 2016.

24. Shi L, Adams MM, Long A, Carter CC, Bennett C, Sonntag WE, Nicolle MM, Robbins M, D'Agostino R and Brunso-Bechtold JK: Spatial learning and memory deficits after whole-brain irradiation are associated with changes in NMDA receptor subunits in the hippocampus. Radiat Res 166: 892-899, 2006. 
25. Semmler A, Garbe S, Moskau S, Frisch C, Eter N, Schlegel U and Linnebank M: An efficient method for fractionated whole rodent brain radiation. Neurol Res 35: 355-359, 2013.

26. Xu M, Fan Q, Zhang J, Chen Y, Xu R, Chen L, Zhao P and Tian Y: NFAT3/c4-mediated excitotoxicity in hippocampal apoptosis during radiation-induced brain injury. J Radiat Res 58: 827-833, 2017.

27. Li YQ, Cheng Z and Wong S: Differential apoptosis radiosensitivity of neural progenitors in adult mouse hippocampus. Int J Mol Sci 17: E97, 2016.

28. Tada E, Parent JM, Lowenstein DH and Fike JR: X-irradiation causes a prolonged reduction in cell proliferation in the dentate gyrus of adult rats. Neuroscience 99: 33-41, 2000.

29. Hassan HA, Hafez HS and Goda MS: Mentha piperita as a pivotal neuro-protective agent against gamma irradiation induced DNA fragmentation and apoptosis: Mentha extract as a neuroprotective against gamma irradiation. Cytotechnology 65: 145-156, 2013.

30. Zhang Y, Cheng Z, Wang C, Ma H, Meng W and Zhao Q: Neuroprotective effects of kukoamine a against radiation-induced rat brain injury through inhibition of oxidative stress and neuronal apoptosis. Neurochem Res 41: 2549-2558, 2016.

31. Derdikman D and Moser EI: A manifold of spatial maps in the brain. Trends Cogn Sci 14: 561-569, 2010.

32. Preston AR and Eichenbaum H: Interplay of hippocampus and prefrontal cortex in memory. Curr Biol 23: R764-R773, 2013

33. Monje ML and Palmer T: Radiation injury and neurogenesis. Curr Opin Neurol 16: 129-134, 2003.

34. Hamani C, Stone SS, Garten A, Lozano AM and Winocur G: Memory rescue and enhanced neurogenesis following electrical stimulation of the anterior thalamus in rats treated with corticosterone. Exp Neurol 232: 100-104, 2011.

35. Schurr A: Neuroprotection against ischemic/hypoxic brain damage: Blockers of ionotropic glutamate receptor and voltage sensitive calcium channels. Curr Drug Targets 5: 603-618, 2004.

36. Li H, Yang X, Shi W, Ma Z, Feng G, Wang Q, Shen L and Xie C: Protective effects of nimodipine on cerebrovascular function in chronic alcoholic encephalopathy. Int J Mol Med 33: 201-208, 2014.
37. Koskimäki J, Matsui N, Umemori J, Rantamäki T and Castrén E: Nimodipine activates TrkB neurotrophin receptors and induces neuroplastic and neuroprotective signaling events in the mouse hippocampus and prefrontal cortex. Cell Mol Neurobiol 35: 189-196, 2015.

38. Ma B and Zhang J: Nimodipine treatment to assess a modified mouse model of intracerebral hemorrhage. Brain Res 1078: 182-188, 2006

39. Li XM, Yang JM, Hu DH, Hou FQ, Zhao M, Zhu XH, Wang Y, Li JG, Hu P, Chen L, et al: Contribution of downregulation of L-type calcium currents to delayed neuronal death in rat hippocampus after global cerebral ischemia and reperfusion. J Neurosci 27: 5249-5259, 2007.

40. Murphy TH, Worley PF and Baraban JM: L-type voltage-sensitive calcium channels mediate synaptic activation of immediate early genes. Neuron 7: 625-635, 1991.

41. Bito H, Deisseroth K and Tsien RW: CREB phosphorylation and dephosphorylation: $\mathrm{A} \mathrm{Ca}(2+)$ - and stimulus duration-dependent switch for hippocampal gene expression. Cell 87: 1203-1214, 1996.

42. West AE, Chen WG, Dalva MB, Dolmetsch RE, Kornhauser JM, Shaywitz AJ, Takasu MA, Tao X and Greenberg ME: Calcium regulation of neuronal gene expression. Proc Natl Acad Sci USA 98: 11024-11031, 2001.

43. Roth KA and D'Sa C: Apoptosis and brain development. Ment Retard Dev Disabil Res Rev 7: 261-266, 2001.

44. Love S: Apoptosis and brain ischaemia. Prog Neuropsychopharmacol Biol Psychiatry 27: 267-282, 2003.

45. Shi J, Miles DK, Orr BA, Massa SM and Kernie SG: Injury-induced neurogenesis in Bax-deficient mice: Evidence for regulation by voltage-gated potassium channels. Eur J Neurosci 25: 3499-3512, 2007. 